normal over a month later. Rechallenge was not performed.

- Case 2-A 75 year old woman, the sister of the first patient and also with no history of allergy or autoimmune disease, was admitted in June 1986 with cutaneous purpura. She had a severe thrombocytopenia $\left(24 \times 10^{9} / 1\right)$, and a bone marrow aspirate showed nothing abnormal. Her only medication was captopril ( $25 \mathrm{mg} / \mathrm{day})$, which she had started taking in April. After withdrawal of captopril the platelet count returned to normal $\left(194 \times 10^{\circ} / 1\right.$ by August 1986$)$. HLA typing was A2 B8 DR3/A3 B14 DR-. No rechallenge was performed.

In both cases the score for drug induced thrombocytopenia was high (C2 S2), leading us to implicate the angiotensin converting enzyme inhibitors. Three points are worth underlining. Firstly, the haematological side effect in both cases was thrombocytopenia alone. Secondly, both sisters shared the B8 DR3 haplotype. In patients with rheumatoid arthritis adverse effects induced by gold salts, particularly haematological effects, are commoner in patients with B8 or DR3 allotypes. 5 Thirdly, the possible mechanism seems to be immunoallergic; in such a situation, and in contrast to leucopenia induced by captopril, rechallenge, even with small doses, is dangerous. These cases thus raise the possibility of a familial immunogenetic background that predisposes to drug induced side effects.

1 Case DB, Whitman H, Laragh JM, Spiera H. Successful low dose captopril rechallenge following drug induced leucopenia. Lancet $1981 ; \mathrm{i}: 1362$.

2 Al Matri A, Larabi MS, Kechrid C, Belkahia C, Ben Ayed H. Fatal bone marrow suppression associated with captopril. BrMed f 1981;283:27.

3 Walsh KP, Branagan J, Walsh MS. Reversible severe thrombocytopenia associated with captopril therapy. Ir Med f 1986; 79:43-4.

4 Khan MA, Bashi SA, Noah MS. Captopril associated isolated thrombocytopenia. Ir Med f 1987;80:101

5 Solal-Celigny P, Benichou C, Boivin P, et al. Assessment criteria for drug induced granulocytic or platelet cytopenia: results of consensus meeting. Nouv Rev Fr Hematol 1987;29:265.

6 Adachi JD, Bensen WG, Singal DP, et al. Gold induced thrombocytopenia, platelet associated IgG and HLA typing in three patients. F R heumatol 1984;11:355-7.

\section{Fetal tachyarrhythmia and maternal antidepressant treatment}

Messrs A PRENTICE and R BROWN (Department of Obstetrics and Gynaecology, North Tees General Hospital, Stockton on Tees TS19 8PE) write: Tricyclic antidepressants taken in overdose can cause tachyarrhythmias in adults. We report a case in which a fetal tachyarrhythmia was believed to be related to maternal tricyclic treatment.

The patient was a 26 year old para $1+1$. After her first full term pregnancy she developed postnatal depression, which required drug treatment. She had no other relevant history. During the first trimester of her current pregnancy she developed a respiratory tract infection, which was treated with penicillin. She then complained of being depressed and was started on dothiepin hydrochloride, initially $50 \mathrm{mg}$ daily. This was increased to $75 \mathrm{mg}$ daily when she was about 16 weeks pregnant and subsequently reduced to 50 and $25 \mathrm{mg}$ daily at 30 and 34 weeks respectively. The general practitioner intended to discontinue treatment before term. An ultrasound scan performed shortly after 18 weeks confirmed gestational age and detected no abnormalities. No problems were detected at 28,30 , and 34 weeks, in particular no abnormalities of the fetal heart rate.

By 37 weeks there had been little growth over the preceding three weeks and her weight had remained static. On auscultation the fetal heart was irregular with a rate of over 180 beats $/ \mathrm{min}$. An ultrasound scan showed a normally grown fetus; the tachyarrhythmia was confirmed and there was no evidence of cardiac failure. She was admitted to the antenatal ward for observation. Over the next three days auscultation showed episodes of fetal tachyarrhythmia. Cardiotocographs performed at other times showed a normal baseline rate. The dothiepin was stopped. The frequency and the duration of the tachyarrhythmias decreased and within four days no abnormalities of the fetal heart rate were detected. At subsequent reviews in the antenatal clinic no abnormalities were noted on auscultation or on cardiotocography. The patient delivered a live male infant $(2980 \mathrm{~g})$ at term. Labour was uneventful, and the Apgar scores were 9 at 1 minute and 10 at 10 minutes. No abnormalities were detected on examination.

Dothiepin hydrochloride is not contraindicated in pregnancy, although there is no evidence of its safety and the datasheet advises that it should be used during pregnancy only "if there are compelling reasons.'

Tachycardia is a well recognised side effect of the tricyclic drugs, as are other disturbances of cardiac rhythm. In this case we presume that the fetal arrhythmia was related to maternal drug reatment: cessation of the treatment resulted in resolution of the episodes of fetal tachyarrhythmia. A Medline search and inquiries to both the drug's manufacturer and the Committee on Safety of Medicines disclosed no other cases. As fetal tachycardias may result in in utero cardiac failure al doctors should be aware of the potential consequences of prescribing tricyclic antidepressants to pregnant women. The advice of the datasheet to use these drugs in pregnancy only if there are compelling reasons should be heeded.

1 Maxwell DJ, Crawford DC, Curry PVM, Tynan MJ, Allan LD.
Obstetric importance, diagnosis, and management of fetal tachycardias. Br.Med f 1988;297:107-10.

\section{Acute pancreatitis associated with infusion of erythromycin lactobionate}

Dr Christopher R E HaWksworth (Department of Orthopaedic Surgery, Monklands District General Hospital, Airdrie ML6 0JS) writes: Acute pancreatitis is occasionally precipitated by drugs. I report a case that followed infusion of erythromycin lactobionate.

A 22 year old woman was admitted with suspected septic arthritis of the left hip. She felt unwell, had a temperature of $38^{\circ} \mathrm{C}$, and had been limping for a week. Hip movements were painfu and restricted. There was tenderness over the hip, but all other joints were unaffected. There was no family history of arthritides. Plain radiographs of the hip, full blood count, and serum calcium phosphate, and alkaline phosphatase values were all normal, but the erythrocyte sedimentation rate was $39 \mathrm{~mm}$ in the first hour. Blood cultures proved sterile.

As she claimed to be allergic to penicillins erythromycin and fucidic acid were prescribed. Since the fucidic acid was not immediately available treatment was started with an infusion of erythromycin lactobionate (Abbott Laboratories) $2 \mathrm{~g}$ in $500 \mathrm{ml}$ of isotonic saline to run over one hour Soon after the infusion started the patient began to feel nauseated and within 40 minutes had developed severe epigastric pain, which radiated to her back. The infusion was stopped. Examination of the abdomen showed nothing abnormal apar from acute epigastric tenderness. Full blood coun and urea and electrolyte measurements were normal, but serum amylase activity was raised $(610 \mathrm{IU} / \mathrm{l})$. The pain settled with appropriate analgesia but recurred later that night, when an amylase activity of $5240 \mathrm{IU} / \mathrm{l}$ confirmed pancreatitis. Blood glucose, calcium, urea, and electrolyte concentrations and arterial blood gas pressures were all normal. There was only minimal disturbance of liver function tests.
She was managed conservatively with nasogastric aspiration, intravenous fluids, and analgesia as needed. Clinically she improved and within five days her blood biochemistry profile had returned to normal. She denied any recent viral illness or alcohol abuse and had not been taking any other medication. There was no history of pancreatitis or biliary disease. The liver, pancreas, and biliary tract were normal on ultrasonography. The suspected hip infection resolved with gentamicin and at three months she had had no further episodes of hip or pancreatic pain

Drug associated pancreatitis is rare.' There have been no published reports linking erythromycin infusion with pancreatitis and the manufacturer knows of no cases. Erythromycin can cause ab dominal pain, jaundice, and hepatocellular toxicity (prescribing information, Abbott Laboratories) and the cramp-like abdominal pains are relieved by antispasmodic agents, suggesting a direct action on smooth muscle in the gut. ' If this were so spasm of the sphincter of Oddi and subsequent bile reflux into the pancreatic duct may have initiated pancreatitis in this case.

Serious complications from drug induced pancreatitis are rare," and it should have been possible to confirm the causal relation between the drug infusion and the pancreatitis by rechallenge. As alternative drugs were available to treat the initial complaint we did not feel this could be justified.

1 Mallory A, Kern F. Drug induced pancreatitis: a critical review. Gastroenterolog 1980; $78: 813-20$.

2 Putzi R, Blaser J, Luthy R, et al. Side effects due to the intravenous infusion of erythromycin lactobionate. Infection 983;11:161-3.

3 Steinberg WM. Acute drug and toxin induced pancreatitis. Hospital Practice 1985;20:95-105.

\section{An allergy to Marmite?}

Dr NIGEL HIGSON (Hove BN3 3DX) writes: Dr Janice Main and colleagues implicate an antibody to bakers' yeast in the development of Crohn's disease.' Yeast may also be implicated in the development of other immune responses. I describe here a case of allergy to a food containing yeast extract (Marmite) which does not seem to have been described in the medical journals (a Medline search revealed no references), yet it seems a common and frightening occurrence, particularly in very young children.

The patient was a 15 month child who apart from having Down's syndrome and an atrioventricular defect was well at the time and had no history of allergy. Marmite was given thinly spread on buttered white bread. Within five minutes mild angio-oedema of the lips and periorbital tissues had occurred. This caused no difficulty in breathing and subsided within 60 minutes. The link with Marmite was not recognised and some days later the child was again given some on bread. This time a more dramatic reaction occurred with a greater degree of angio-oedema of the mouth and periorbital tissues such that the child exhibited some difficulty in respiration. Most of the oedema settled again within 60 minutes and the child did not require drug treatment. On talking to other parents of young children I found that several other children exhibit similar reactions to Marmite and, although I know of no major anaphylactic reaction occurring, the angio-oedema is sufficiently severe to cause concern to the parent. Should such products be promoted as a weaning food? Some health visitors advise mothers to put Marmite on their nipples to break the child's breast feeding habit; in a susceptible child this action might possibly be fatal.

I Main J, Mlckenzie H, Yeoman GR, et al. Antihede to Saccharome 1988:297:1105-6. 290ctober. 